5. H. Arai and K. Kohzu, A bidirectional notch antenna, In: IEEE Antennas and Propagation Society International Symposium, Vol. 1, Baltimore, MD, 1996, pp. 42-45.

6. H. Arai, K. Kohzu, and T. Mukaiyama, Bi-directional notch antenna with parasitic elements for tunnel booster system, In: IEEE Antennas and Propagation Society International Symposium, Vol. 4, Montreal, Canada, 1997, pp. 2218-2221.

7. J.D. Dyson, The equiangular spiral antenna, IRE Trans Antennas Propag 7 (1959), 181-187.

8. K. Cho and T. Hori, Bidirectional rod antenna composed of narrow patches, In: IEEE Antennas and Propagation Society International Symposium, Vol. 1, Seattle, WA, 1994, 174-177.

9. C. Phongcharoenpanich, T. Sroysuwan, P. Wounchum, S. Kosulvit, and M. Krairiksh, Theory and experiment of an antenna using a probe excited rectangular ring, In: IEEE Antennas and Propagation Society International Symposium, Columbus, OH, Vol. 3, 2003, 737-740.

(C) 2013 Wiley Periodicals, Inc.

\section{EFFECTIVE DIELECTRIC CONSTANT OF TOP GROUNDED COPLANAR WAVEGUIDE ON LIQUID CRYSTAL SUPERSTRATE}

Senad Bulja, ${ }^{1}$ Dariush Mirshekar-Syahkal, ${ }^{2}$ Richard James, ${ }^{3}$ Sally E. Day, ${ }^{3}$ and F. Aníbal Fernández ${ }^{3}$

${ }^{1}$ Bell Labs Ireland, Blanchardstown Industrial Park, Dublin 15, Ireland

${ }^{2}$ School of Computer Science and Electronic Engineering, University of Essex, Colchester CO4 3SQ, United Kingdom ${ }^{3}$ Department of Electronic and Electrical Engineering, University College London, Torrington Place, London WC1E 7JE, United Kingdom; Corresponding author: senad.bulja@alcatel-lucent.com

Received 26 September 2012

ABSTRACT: The effective dielectric constant of the top grounded coplanar waveguide with a liquid crystal $(L C)$ superstrate for phase shifting applications is investigated in the frequency range of 30-60 GHz. Two nematic LC mixtures, namely E7 and MDA-00-3506, are used as the superstrate. The measurements show that MDA-00-3506 offers higher values of phase shift per millimeter than its E7 counterpart. In particular, the MDA-00-3506 provides $3.14^{\circ} / \mathrm{mm}$, whereas E7 gives $2.79^{\circ} / \mathrm{mm}$ at 60 $\mathrm{GHz}$. The results of the dielectric constants from measurement and computer modeling are found to agree to within 5\%. For the modeling, a comprehensive finite element package predicting the local alignment of LC molecules and effective dielectric constant at different bias voltages and frequencies are used. (C) 2013 Wiley Periodicals, Inc. Microwave Opt Technol Lett 55:1416-1418, 2013; View this article online at wileyonlinelibrary.com. DOI 10.1002/mop.27564

Key words: $C P W$; liquid crystal; finite element method; mm-wave devices

\section{INTRODUCTION}

The abundance of a widely available spectrum between 30 and $60 \mathrm{GHz}$ (in mm-wave region) offers potential for the development of high data rate, short-range wireless communications [1].

Nematic liquid crystals (LCs) are relatively cheap and their dielectric constants, which have a tensor form, can be controlled by an external electric or magnetic field. This property of nematic LCs has already been exploited for the development of tunable antennas [2] and phase shifters [3].

The choice of a suitable LC for a particular mm-wave application depends on many factors, however, by far the most influential one is the dielectric anisotropy, $\Delta \varepsilon=\varepsilon_{\|}-\varepsilon_{\perp}$, as it directly influences the tunability of an LC-based device.

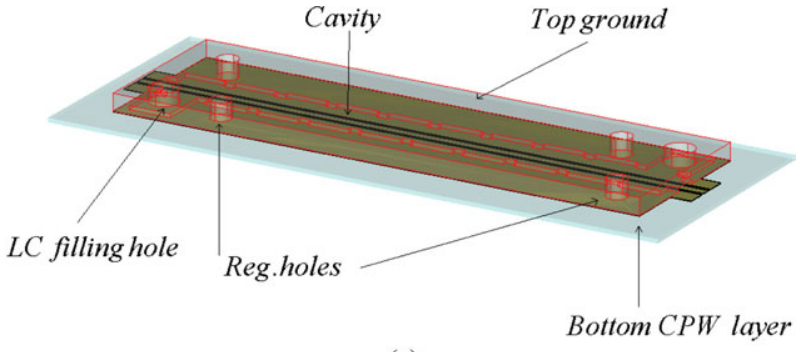

(a)

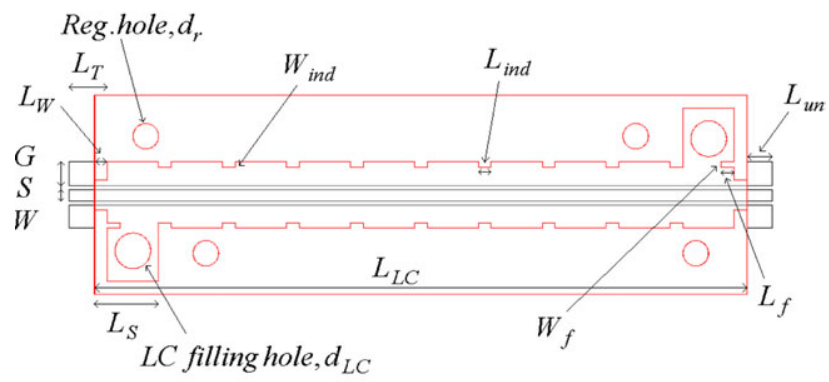

(b)

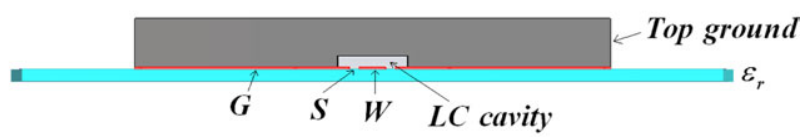

(c)

Figure 1 Device structure for measurement of effective dielectric constant of top grounded CPW with LC superstrate: (a) perspective, (b) top, and (c) cross-section views (not to scale). [Color figure can be viewed in the online issue, which is available at wileyonlinelibrary.com]

LC-based mm-wave devices can be developed using various planar transmission lines including the microstrip line and coplanar waveguide (CPW). However, the implementation of an LC layer as a substrate in a microstrip line and integrating this line with the rest of microstrip lines in a circuit are difficult, while an LC superstrate can be realized in a part of a CPW circuit with relative ease.

In this article, the variation of the effective dielectric constant of the top grounded CPW loaded with an LC superstrate with frequency and LC bias voltage is investigated, and its use in phase shifting applications is outlined. The frequency range considered is 30-60 GHz. In the investigation, two different LCs, known as E7 and MDA-00-3506, are used. The measured effective dielectric constants are then compared with those obtained from a finite element modeling developed by the authors for the analysis and design of LC-based planar structures [4].

\section{MEASUREMENT DEVICE AND TECHNIQUE}

The top grounded CPW with the LC cell as its superstrate is shown in Figure 1. For the dielectric characterization purpose, a length of the top grounded CPW with the LC cell (superstrate) is terminated into two short sections of ungrounded CPWs. In fact, the use of CPW electrodes allows for the measurements of $S$-parameters using a probe station at different bias voltages supplied through a wideband bias tee. The rotation of the LC molecules in the LC cell is achieved by applying low frequency and low voltage across the electrodes of the ungrounded CPW [4], which is in contrast to the magnetic field biasing method used in Ref. 5. 


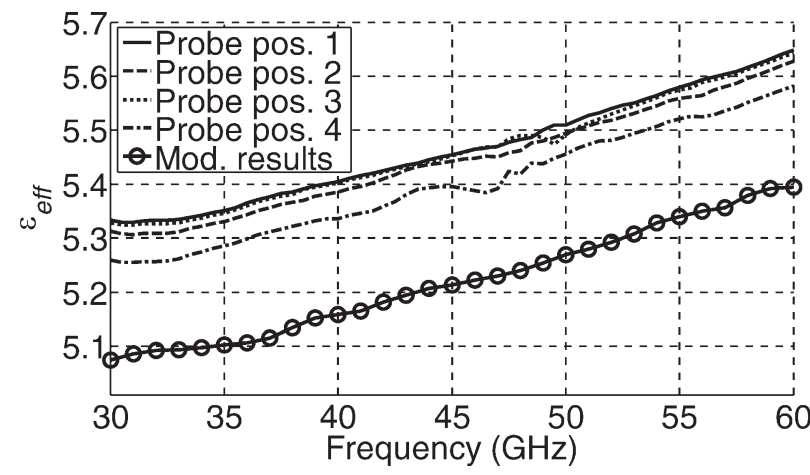

Figure 2 Measured effective dielectric constants of top grounded $\mathrm{CPW}$ with $\mathrm{E} 7$ superstrate biased at $0 \mathrm{~V}$ for four probe positions and result from modeling

As shown in Figure 1, all the electrodes of the CPWs are patterned on a continuous dielectric substrate. The dielectric constant of the substrate is $\varepsilon_{\mathrm{r} 1}=9.8$ and its thickness is $h_{1}=$ $381 \mu \mathrm{m}$. The ungrounded CPW terminations are $50 \Omega$, and the electrode dimensions of the CPWs are $G=450 \mu \mathrm{m}, S=80$ $\mu \mathrm{m}$, and $W=200 \mu \mathrm{m}$. The patterned U-shape conductor block, Figure 1, providing the housing for the LC superstrate is copper and is chemically etched to a depth of $h_{\mathrm{c}}=80 \mu \mathrm{m}$.

The lengths of the top grounded CPW and normal CPW sections are $L_{\mathrm{LC}}=12,750 \mu \mathrm{m}$ and $L_{\mathrm{un}}=500 \mu \mathrm{m}$, respectively. The distance between the CPW center conductor to a CPW ground, $S$, is the same as the depth of the LC conductor housing, $h_{\mathrm{c}}$, which ensures approximately equal field distribution between the center conductor and CPW grounds on the dielectric substrate and between the center conductor and the top ground plane. The small patterned periodical indentations with a spatial period of $L_{\mathrm{S}}=$ $1250 \mu \mathrm{m}$ evident in Figure 1 along the edges of the U-shape conductor housing prevent the build up of standing waves in the housing within the frequency band of interest. The rest of the dimensions shown in Figure 1 are $L_{\mathrm{W}}=250 \mu \mathrm{m}, W_{\text {ind }}=$ $100 \mu \mathrm{m}, W_{\mathrm{f}}=100 \mu \mathrm{m}$, and $L_{\mathrm{f}}=250 \mu \mathrm{m}$. Additionally, the diameter of the registration holes is $d_{\mathrm{r}}=500 \mu \mathrm{m}$, whereas the diameter of the LC filling holes is $d_{\mathrm{LC}}=700 \mu \mathrm{m}$.

To extract the parameters (effective dielectric constant, etc.) of the top grounded CPW with the LC superstrate, Figure 1, the effects of the two transitions at the two ends of this CPW section must be accounted for first. This is done by using a modified thru-line two tier de-embedding procedure developed by the authors in Ref. 6. The extraction of the effective dielectric con-

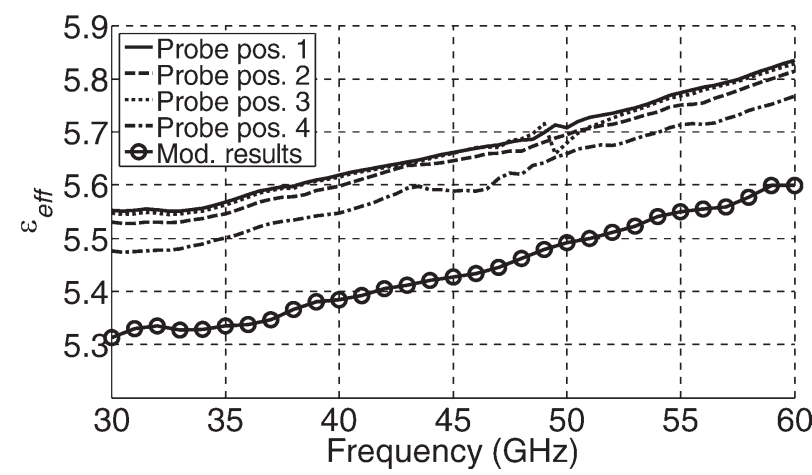

Figure 3 Measured effective dielectric constants of top grounded CPW with E7 superstrate biased at $11 \mathrm{~V}$ for four probe positions and result from modeling

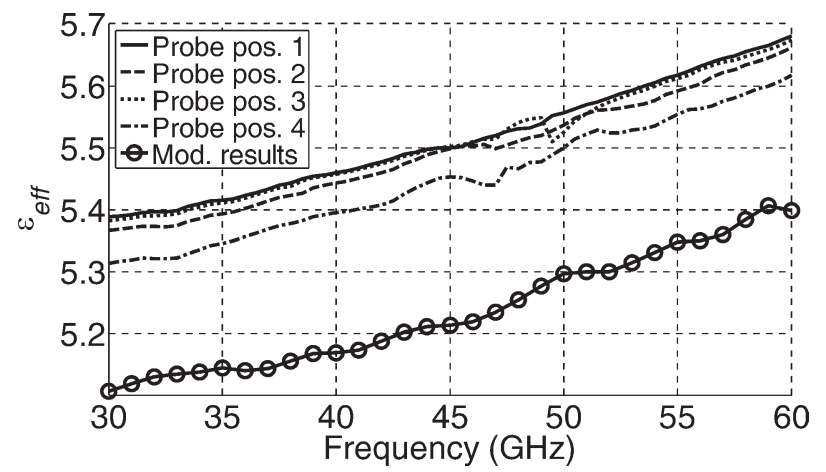

Figure 4 Measured effective dielectric constants of top grounded CPW with MDA-00-3506 superstrate biased at $0 \mathrm{~V}$ for four probe positions and result from modeling

stants of nematic LCs under various voltages is then achieved using the following matrix expression

$$
[T]_{\text {meas }}=[T]_{\mathrm{tr}} \cdot[T]_{\mathrm{LC}} \cdot[T]_{\mathrm{revtr}}
$$

where $[T]_{\text {meas }}$ is the overall measured transmission matrix of the device in Figure $1,[T]_{\mathrm{tr}}$ and $[T]_{\text {revir }}$ are the transmission matrices for the transition in forward and reverse directions, respectively, and $[T]_{\mathrm{LC}}$ is the transmission matrix of the CPW with LC superstrate, given by

$$
[T]_{\mathrm{LC}}=\left[\begin{array}{cc}
\mathrm{e}^{-\gamma L_{\mathrm{L}}} & 0 \\
0 & \mathrm{e}^{\gamma L_{\mathrm{L}}}
\end{array}\right]
$$

In Eq. (2), $\gamma=\alpha_{\mathrm{eff}}+j \beta_{\mathrm{eff}}$ is the complex propagation constant, obtained by solving Eq. (1). Considering the LCs used are of low-loss, the effective dielectric constant, $\varepsilon_{\text {eff }}$, of the top grounded $\mathrm{CPW}$ with the LC substrate is then found to be:

$$
\varepsilon_{\text {eff }}=\left(\frac{\beta_{\text {eff }} \lambda_{0}}{2 \pi}\right)^{2}
$$

where $\lambda_{0}$ is the free space wavelength.

\section{MEASUREMENT RESULTS}

The device in Figure 1 was fabricated using the standard photolithography process. In the fabrication, a commercially available dielectric substrate, TMM 10i from Rogers Duroid [7], was used

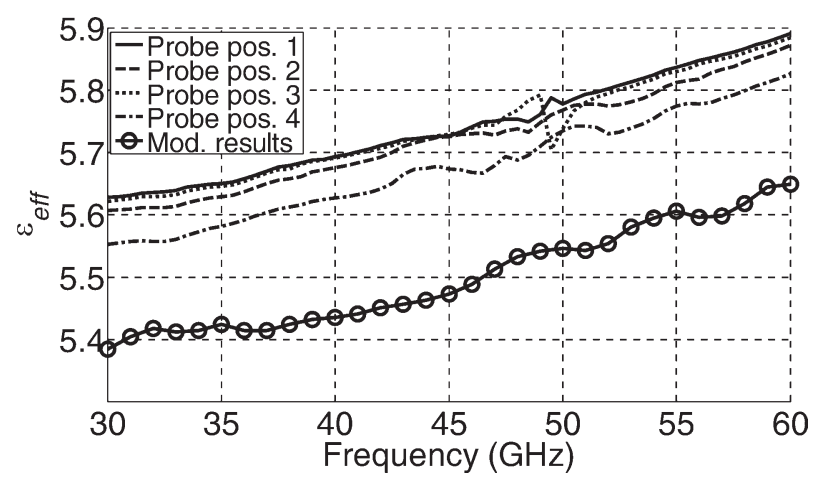

Figure 5 Measured effective dielectric constants of top grounded CPW with MDA-00-3506 superstrate biased at $11 \mathrm{~V}$ for four probe positions and result from modeling 
TABLE 1 Measured Phase Shift per Length of LC-Based CPW Superstrate Structure

\begin{tabular}{lccccc}
\hline & Freq. (GHz) & 30 & 40 & 50 & 60 \\
$\Delta \Phi(\% / \mathrm{mm})$ & E7 & 1.689 & 2.181 & 2.509 & 2.795 \\
& MDA-00-3506 & 1.843 & 2.37 & 2.843 & 3.144
\end{tabular}

on which the CPW electrodes were patterned. The internal surface of the U-shape ground conductor block housing the LC, Figure 1, and the surface of the dielectric substrate with patterned CPW electrodes were spin coated with a thin passivation layer of polyimide to achieve surface planarization. The polyimide covered surfaces were then mechanically rubbed in the direction of wave propagation in the antiparallel fashion. This procedure ensures anchoring of the LC molecules in the direction of rubbing, defining the so-called "ground state" of the LC in the absence of an external voltage bias. Two devices as shown in Figure 1 were fabricated and each filled with one of the LC mixtures, namely, E7 and MDA-00-3506. In the measurements, a slow varying bias signal of frequency $200 \mathrm{~Hz}$ was applied to the LC superstrate through a wideband bias tee. The $S$-parameters of the devices were measured at a bias signal amplitudes of 0 and $11 \mathrm{~V}$ at a temperature of about $23^{\circ} \mathrm{C}$ (room temperature). Due to the uncertainty associated with the landing positions of the CPW probes on the CPW terminals of the device in the measurements, Figure 1, the measurements were repeated four times for each device, and the effective dielectric constants are extracted using Eqs. (1)-(3), Figures 2-5.

The results presented in Figures $2-5$ are for the two devices with the LC biased at $0 \mathrm{~V}$ (ground state) and at $11 \mathrm{~V}$ (fully switched-on state). Examining the results, the following two observations are noteworthy. First, there is some influence of the uncertainty in the landing position of the CPW probes on the measured $\varepsilon_{\text {eff }}$. If it can be assumed that the correct value of the measured $\varepsilon_{\text {eff }}$ is the average of all $\varepsilon_{\text {eff }}$ measured for different landing positions of the CPW probes, then the maximum error of just over $1 \%$ can be attributed to the probe landing position. The second observation relates to some disagreements between the measured $\varepsilon_{\text {eff }}$ and those obtained by the finite element modeling [4] over the concerned frequency range $(30-60 \mathrm{GHz})$. The finite element modeling program uses the electrical properties of the two LCs obtained from a broadband measurement technique developed by the authors [4]. These properties are believed to be accurate to within $1 \%$ in the frequency range of $30-60 \mathrm{GHz}$. Therefore, the maximum error of 5\% observed between the measured and the modeled results is believed to be due to imperfections in the fabrication process.

An important parameter in the design of LC-based devices including phase shifters is the amount of differential phase shift per unit length, $\Delta \Phi$. Table 1 summarizes this value for the investigated LC filled CPW devices across the $30-60 \mathrm{GHz}$ range. It shows that MDA-00-3506 provides larger phase shift.

\section{CONCLUSION}

This article investigated the variation of the effective dielectric constant of the top grounded CPW using an LC superstrate at the frequency range of 30-60 GHz. The effective dielectric constants of two LC filled top grounded CPW are extracted from the $S$-parameters measurements for two different LC mixtures, E7 and MDA-00-3506 at different bias voltages. The modeling and measured results follow each other closely, to within 5\% accuracy. Based on the extracted values of the effective dielectric constants, the amount of phase shift per unit length achieved by the top grounded CPW for each LC mixture was calculated. It was found that the top grounded CPW including MDA-00-3506 offers a superior phase shifting performance of $3.14^{\circ} / \mathrm{mm}$ compared to that of E7, which offers $2.79^{\circ} / \mathrm{mm}$ at $60 \mathrm{GHz}$.

\section{REFERENCES}

1. Federal Communications Commission, Amendment of parts 2, 15 and 97 of the commission's rules to permit use of radio frequencies above $40 \mathrm{GHz}$ for new radio applications, Federal Communications Commission, Washington, DC (1995). Available at ftp://ftp.fcc.gov/ pub/Bureaus/Engineering_Technology/Orders/1995/fcc95499.txt.

2. N. Martin, P. Laurent, C. Person, P. Gelin, and F. Huret, Size reduction of a liquid crystal-based, frequency-adjustable patch antenna, In: Proceedings of the 34th European Microwave Conference, Amsterdam, The Netherlands, October 2004, pp. 825-828.

3. S. Bulja, D. Mirshekar-Syahkal, M. Yazdanpanahi, R. James, F.A. Fernandez, and S.E. Day, $60 \mathrm{GHz}$ reflection type phase shifter based on liquid crystal, In: Proceedings of IEEE RWS 2010, New Orleans, USA, 10-14 January 2010, pp. 697-699.

4. S. Bulja, D. Mirshekar-Syahkal, R. James, F.A. Fernandez, and S.E. Day, Measurement of dielectric properties of nematic liquid crystals at millimetre wavelength, IEEE Trans Microwave Theory Tech 58 (2010), 3493-3501.

5. A. Penirschke, C. Damm, P. Scheele, M. Wittek, C. Weil, and R. Jakoby, Broad-band microwave characterization of liquid crystals using a temperature-controlled coaxial transmission line, IEEE Trans Microwave Theory Tech 53 (2005), 1937-1944.

6. S. Bulja and D. Mirshekar-Syahkal, Novel wide-band transition between coplanar waveguide and microstrip line, IEEE Trans Microwave Theory Tech 58 (2010), 1851-1857.

7. Available at: http://www.rogerscorporation.com, 2010.

(C) 2013 Wiley Periodicals, Inc.

\section{AN ANNULAR-RING SLOT ANTENNA FOR CP OPERATION}

Ajay Kumar, ${ }^{1}$ A. K. Gautam, ${ }^{1}$ and Binod Kr. Kanaujia ${ }^{2}$

${ }^{1}$ Department of Electronics \& Communication Engineering, G.B. Pant Engineering College, Pauri Garhwal, Uttarakhand 246 194, India; Corresponding author: drakgautam@ieee.org

${ }^{2}$ Department of Electronics \& Communication Engineering, Ambedkar Institute of Advanced Communication Technologies \& Research, Delhi 110031, India

\section{Received 27 September 2012}

ABSTRACT: An annular-ring slot antenna with pair of slits and truncated corner for CP operation is proposed. A pair of slits and truncated corners are used to enhance axial ratio bandwidth. The proposed antenna is fabricated and simulated to analyze its radiation characteristics. Simulated results show a good agreement with experimental results. The antenna also shows much larger $C P$ bandwidths than earlier reported results. The measured impedance bandwidth and 3-dB axial ratio bandwidth (ARBW) of the proposed antenna are 94.2 and $73.8 \%$, respectively. The proposed antenna shows consistent return loss and radian pattern for entire frequency band. (C) 2013 Wiley Periodicals, Inc. Microwave Opt Technol Lett 55:14181422, 2013; View this article online at wileyonlinelibrary.com. DOI 10.1002/mop. 27563

Key words: annular-ring slot antenna; axial ratio; bandwidth; circular polarization; microstrip antenna

\section{INTRODUCTION}

Microstrip antennas can be divided into two basic types by structure, namely microstrip patch antenna [1-3] and microstrip slot antenna [4, 5]. Many communication and sensor systems 